\title{
How to Convert the Flavor of a Quantum Bit Commitment
}

\author{
Claude Crépeau ${ }^{1}$, Frédéric Légaré ${ }^{2}$, and Louis Salvail ${ }^{3}$ \\ 1 School of Computer Science, McGill University ${ }^{\dagger}$, crepeau@cs.mcgill.ca \\ 2 ZKLabs $^{\ddagger}$, Zero-Knowledge Systems Inc., frederic@zeroknowledge.com \\ 3 BRICS $^{\S}$, Dept. of Computer Science, University of Århus, salvail@brics.dk
}

\begin{abstract}
In this paper we show how to convert a statistically binding but computationally concealing quantum bit commitment scheme into a computationally binding but statistically concealing QBC scheme. For a security parameter $n$, the construction of the statistically concealing scheme requires $O\left(n^{2}\right)$ executions of the statistically binding scheme. As a consequence, statistically concealing but computationally binding quantum bit commitments can be based upon any family of quantum one-way functions. Such a construction is not known to exist in the classical world.
\end{abstract}

\section{Introduction}

Finding the weakest computational assumptions from which the basic cryptographic primitives can be based upon is important for the theoretical foundations of cryptography. Protocols for secure 2-party computations are usually built from two basic and fundamental cryptographic primitives: Bit commitment and oblivious transfer. Classically, one-way functions are necessary and sufficient for secure bit commitment but not for oblivious transfer unless a major breakthrough is achieved in complexity theory [10]12. This suggests that in classical cryptography, bit commitment is a weaker primitive than oblivious transfer. Bit commitments come in two main flavors: binding but computationally concealing and concealing but computationally binding. Informally, binding means that whatever the committer does, it is impossible to open both 0 and 1 with non-negligible probability of success (this is sometimes called statistically binding). Concealing means that the receiver cannot obtain more than a negligible amount of information about the committed bit (i.e. statistically concealing). The weakest known computational assumption from which bit commitment can be based upon depends on its flavor. Binding but computationally concealing bit commitments can be based upon any one-way function [17/117]. On the other hand, the weakest known assumption for concealing but computationally

\footnotetext{
† Part of this research was funded by Québec's Fonds FCAR and Canada's NSERC.

$\ddagger$ This research was done as part of the M.Sc. requirements at McGill University.

$\S$ Basic Research in Computer Science (www.brics.dk), funded by the Danish National Research Foundation.
} 
binding commitments is the existence of one-way permutations [18. It seems that in the classical world, concealing commitments are more difficult to achieve than binding ones. The two flavors allow for different cryptographic applications. For example, computational zero-knowledge proofs [8]9] can be constructed from binding commitments whereas perfect zero-knowledge arguments 4 use concealing commitments.

In quantum cryptography, computational assumptions are also required for bit commitment and oblivious transfer [15,16 14]. The standard computational assumptions for the quantum case are defined as in the classical case except that they must resist quantum inverters. A quantum one-way function is simply a classical function $f:\{0,1\}^{n} \rightarrow\{0,1\}^{l(n)}$ for which given any $x \in\{0,1\}^{n}, f(x)$ can be efficiently computed by a quantum computer but finding $x^{\prime} \in f^{-1}(y)$ given $y:=f(x)$, (when $x \in_{R}\{0,1\}^{n}$ ) is hard. In [6], a concealing quantum bit commitment scheme is built from any quantum one-way permutation. The resulting scheme, although improving the communication complexity of the known classical protocols, requires the same kind of assumption as in the classical case. In this paper, we show that the computational assumption for concealing quantum bit commitment schemes can be weakened compared to its classical counterpart. Our construction relies upon the QOT protocol for quantum 1-out-of-2 oblivious transfer of Crépeau [5]. The QOT protocol can be seen as a construction of quantum oblivious transfer from a black-box for bit commitment [519]. Therefore and unlike the classical case, there exists a black-box reduction of quantum oblivious transfer to bit commitment.

Our main contribution consists in showing how any statistically binding quantum bit commitment scheme can be transformed into a statistically concealing one. The construction is obtained by using the QOT protocol together with statistically binding but otherwise computationally concealing commitments (these commitments will be called initial commitments in the following). Using the QOT protocol that way, we construct a simple quantum commitment scheme that we show statistically concealing and computationally binding. The construction converts the flavor of the initial commitments after calling them $O\left(n^{2}\right)$ times for $n$ a security parameter. As a byproduct, we show that the QOT protocol is an oblivious transfer that statistically hides one out of the two bits sent and computationally conceals the receiver's selection bit whenever it is used together with statistically binding but computationally concealing commitments instead of perfect commitments given as black-boxes. This extends the security result for the QOT protocol of [519] to the computational case. Our reduction of an adversary for the binding condition of the resulting commitment scheme to an adversary for the concealing condition of the initial commitment is expected polynomial-time black-box. Although quantum information has peculiar behaviors adding complexity to the security proofs of cryptographic protocols, we shall see that using quantum oblivious transfer as a primitive allows to return to an essentially classical situation. This might be of independent interest for the construction and analysis of complex quantum protocols. 
One consequence of our result is that statistically concealing but computationally binding quantum commitment scheme can be based upon any quantum one-way function using Naor's construction [17] from pseudo-random bit generators. Only the ability to send and receive BB84 1] qubits is required in order to get the new flavor. The scheme can therefore be implemented using current technology. Our result gives more evidences that computational security in 2-party quantum cryptography enjoys different properties than its classical counterpart.

Paper's Organization. We introduce tools and definitions in Sect. 2] The protocol by which the flavor of an originally binding but computationally concealing commitment is transformed into a concealing but computationally binding commitment is described in Sect. 3. The security proof of our construction is given in Sect. 4 and Sect. 5 In Sect. 4 we show that the resulting commitment is computationally binding if the original one was computationally concealing. We then prove in Sect. 5 that if the initial commitment scheme is binding then the resulting one is concealing. We finally conclude in Sect.6.

\section{Preliminaries}

\subsection{Tools}

Let $X \sim B(p)$ be a Bernoulli random variable with probability of success $p$ (when $X=1$ ). The following simple argument will be useful:

Hybrid Argument. Let $\mathcal{X}=\left\{X_{1}, X_{2}, \ldots, X_{n}\right\}$ be a set of independent random variables $X_{i} \sim B\left(p_{i}\right)$ for $1 \leq i \leq n$. Then, there exist $1 \leq k<n$ such that,

$$
\left|p_{k+1}-p_{k}\right| \geq \frac{\left|p_{n}-p_{1}\right|}{n}
$$

The result also holds without the absolute values. Later, we shall be given $\mathcal{X}$ without the values of the $p_{i}$ 's but only circuits (quantum or classical) $\mathrm{R}_{i}$ for sampling in each $X_{i} \in \mathcal{X}$ (i.e. $\left.\mathrm{P}\left(\mathrm{R}_{i}=0\right)=p_{i}\right)$ and a guarantee that (1) holds for some $k$. In this scenario, we shall need an algorithm for estimating the $p_{i}$ 's and one for finding $k^{\prime}$ that satisfies a drop similar to (1).

Estimating the $\boldsymbol{p}_{\boldsymbol{i}}$ 's. Let $\mathrm{R}$ be a circuit for sampling in $B(p)$ where $p=q+\frac{1}{p(n)}$, $0 \leq q<1$ is a known constant, and $p(n)$ is a positive polynomial. It is easy to devise an algorithm LowBound(R, $q, n)$ that satisfies (see [13] for the proof and the algorithm):

Lemma 1. For $n$ sufficiently large, LowBound $(\mathrm{R}, q, n)$ returns $\frac{1}{g_{n}}$ such that $\frac{1}{n^{2} p(n)}<\frac{1}{g_{n}} \leq \frac{1}{p(n)}$ except with probability $2^{-\alpha n}, \alpha>0$ and after calling $\mathrm{R}$ an expected $O\left(n^{5} p(n)^{2}\right)$ times. 
Finding a Drop. Let $\mathcal{D}_{m}\left(\frac{1}{p(n)}\right)=\left\{p_{i}\right\}_{i=0}^{m}$ be a family of Bernoulli distributions with unknown parameters $0 \leq p_{i} \leq 1$ for every $0 \leq i \leq m$ and such that $p_{k^{*}}-p_{k^{*}+1} \geq \frac{1}{p(n)}$ for some $0 \leq k^{*}<m$. Let $\mathrm{S}$ be a sampling circuit for $\mathcal{D}$ that given $0 \leq l \leq m$ runs $\mathrm{R}_{m}$ (i.e. $\left.\mathrm{P}(\mathrm{S}(l)=1)=1-\mathrm{P}(\mathrm{S}(l)=0)=p_{l}\right)$. We would like to find $\kappa$ that exhibits a polynomial drop $p_{\kappa}-p_{\kappa+1}$ similar to $p_{k^{*}}-p_{k^{*}}$. It is not difficult to find an algorithm FindDrop that finds $\kappa$ (using the sampling circuit $\mathrm{S}$ as a black-box) such that (see [13] for the proof and the algorithm):

Lemma 2. Given a family of Bernoulli distributions $\mathcal{D}_{m}\left(\frac{1}{p(n)}\right)=\left\{p_{i}\right\}_{i=1}^{m}$ with sampling circuit $\mathrm{S}$ such that $p_{k^{*}}-p_{k^{*}+1} \geq \frac{1}{p(n)}$ for some $0 \leq k^{*} \leq m-1$, algorithm FindDrop $\left(\mathrm{S}, \frac{1}{p(n)}, n\right)$ returns $\kappa$ such that $p_{\kappa}-p_{\kappa+1} \geq \frac{1}{2 p(n)}$ except with negligible probability $2^{-\alpha n}, \alpha>0$ and after calling $\mathrm{S}$ at most $O\left(m^{2} n p(n)^{2}\right)$ times.

\subsection{Notations and Model of Computation}

For simplicity, we shall often drop the security parameters associated with protocol executions. When protocols and adversaries are modeled as circuits they should be understood as infinite families of circuits, one circuit for each possible values of the security parameters. We write $\operatorname{poly}(n)$ for the set of all positive polynomials.

Let $\mathcal{H}_{n}$ denote a $n$-dimensional Hilbert space, that is a complete inner product vector space over the complex numbers. The basis $\{|0\rangle,|1\rangle\}$ denotes the computational or rectilinear or "+" basis for $\mathcal{H}_{2}$. When the context requires, we write $|b\rangle_{+}$to denote the bit $b$ in the rectilinear basis. The diagonal basis, denoted " $\times$ ", is defined as $\left\{|0\rangle_{\times},|1\rangle_{\times}\right\}$where $|0\rangle_{\times}=\frac{1}{\sqrt{2}}(|0\rangle+|1\rangle)$ and $|1\rangle_{\times}=\frac{1}{\sqrt{2}}(|0\rangle-|1\rangle)$. The states $|0\rangle,|1\rangle,|0\rangle_{\times}$and $|1\rangle_{\times}$are the four BB84 states. For any $x \in\{0,1\}^{n}$ and $\theta \in\{+, \times\}^{n}$, the state $|x\rangle_{\theta}$ is defined as $\otimes_{i=1}^{n}\left|x_{i}\right\rangle_{\theta_{i}}$ where $\otimes$ denotes the tensor product. An orthogonal (or von Neumann) measurement of a quantum state in $\mathcal{H}_{m}$ is described by a set of $m$ orthogonal projections $\mathcal{M}=\left\{\mathbb{P}_{i}\right\}_{i=1}^{m}$ acting in $\mathcal{H}_{m}$ thus satisfying $\sum_{i} \mathbb{P}_{i}=\mathbb{1}_{m}$ where $\mathbb{1}_{m}$ denotes the identity operator in $\mathcal{H}_{m}$. Each projection or equivalently each index $i \in\{1, \ldots, m\}$ is a possible classical outcome for $\mathcal{M}$.

We model quantum algorithms by quantum circuits built out of a universal set of quantum gates $\mathcal{U G}=\left\{\right.$ CNot, $\left.\mathrm{H}, \mathrm{R}_{\mathbb{Q}}\right\}$, where CNot denotes the controlledNOT, $H$ the one qubit Hadamard gate, and $R_{\mathbb{Q}}$ is an arbitrary one qubit non-trivial rotation specified by a matrix containing only rational numbers [2]. The timecomplexity of a quantum circuit $C$ is the number of elementary gates $\|C\|_{\mathcal{U G}}$ in $\mathcal{C}$. In addition to the set of gates $\mathcal{U G}$, a quantum circuit is allowed to perform one kind of von Neumann measurement: $\mathcal{M}_{+}=\left\{\mathbb{P}_{0}^{+}, \mathbb{P}_{1}^{+}\right\}$where $\mathbb{P}_{0}^{+}=|0\rangle\langle 0|$ and $\mathbb{P}_{1}^{+}=|1\rangle\langle 1|$ are the two orthogonal projections of the computational basis. $\mathcal{M}_{+}$ is sometimes called the measurement in the rectilinear or computational basis. Another von Neumann measurement used by the receiver in the BB84 quantum coding scheme is the measurement in the diagonal basis $\mathcal{M}_{\times}=\left\{\mathbb{P}_{0}^{\times}, \mathbb{P}_{1}^{\times}\right\}$for $\mathbb{P}_{0}^{\times}=\frac{1}{2}(|0\rangle+|1\rangle)(|0\rangle+|1\rangle)^{\dagger}$ and $\mathbb{P}_{1}^{\times}=\frac{1}{2}(|0\rangle-|1\rangle)(|0\rangle-|1\rangle)^{\dagger}$ where ${ }^{\dagger}$ denotes the transposed-complex conjugate operator. The Hadamard gate $\mathrm{H}$ is sufficient to 
build measurement $\mathcal{M}_{\times} \in \mathcal{U G}$ from $\mathcal{M}_{+}$since $\mathcal{M}_{\times}=\left\{\mathrm{HP}_{0}^{+} \mathrm{H}^{\dagger}, \mathrm{HP}_{1}^{+} \mathrm{H}^{\dagger}\right\}$. For $x \in$ $\{0,1\}^{n}$ and $\beta \in\{+, \times\}^{n}$ we write $\mathbb{P}_{x}^{\beta} \equiv \otimes_{i=1}^{n} \mathbb{P}_{x_{i}}^{\beta_{i}}$. If $|\Psi\rangle \in H_{A} \otimes H_{B}$ is a composite quantum state, we write $\mathbb{P}_{x}^{A}|\Psi\rangle$ (i.e. $\mathbb{P}_{x}^{A} \otimes \mathbb{1}^{B}|\Psi\rangle$ ) for the projector applied to the registers in $H_{A}$ along the state $|x\rangle$ for $x \in\{0,1\}^{\operatorname{Dim}\left(H_{A}\right)}$. The classical output $L(|\Psi\rangle)$ of circuit $L$ is the classical outcomes of all von Neumann measurements $\mathcal{M}_{+}$taking place during the computation $L|\Psi\rangle$. If the circuit $L$ accepts two input states of the form $\left|\Psi_{0}\right\rangle \otimes\left|\Psi_{1}\right\rangle$ we may write similarly $L\left(\left|\Psi_{0}\right\rangle,\left|\Psi_{1}\right\rangle\right)$ for the classical output.

A 2-party quantum protocol is a pair of interactive quantum circuits $(A, B)$ applied to some initial product state $\left|x_{A}\right\rangle^{A} \otimes\left|x_{B}\right\rangle^{B}$ representing $A$ 's and $B$ 's inputs to the protocol neglecting to write explicitly the states of $A$ 's and $B$ 's registers that do not encode their respective input to the protocol (thus all in initial states $|0\rangle)$. Also, we shall often write $\left|x_{A}\right\rangle^{A}\left|x_{B}\right\rangle^{B}$ for the product state without explicitly writing the tensor product $\otimes$. Since communication takes place between $A$ and $B$, the complete circuit representing one protocol execution may have quantum gates in $A$ and $B$ acting upon the same quantum registers. We write $A \odot B$ for the complete quantum circuit when $A$ is interacting with $B$. The final composite state $\left|\Psi_{\text {final }}\right\rangle$ obtained after the execution is then written as $\left|\Psi_{\text {final }}\right\rangle=(A \odot B)\left|x_{A}\right\rangle^{A}\left|x_{B}\right\rangle^{B}$.

\subsection{Cryptographic Primitives}

The two relevant quantum primitives we shall use heavily in the following are quantum bit commitment and quantum oblivious transfer. They are defined as straightforward quantum generalizations of their classical counterparts.

Quantum Bit Commitment. A quantum bit commitment scheme is defined by two quantum protocols $\left(\left(C^{A}, C^{B}\right),\left(O^{A}, O^{B}\right)\right)$ where $\left(C^{A}, C^{B}\right)$ is a pair of interactive quantum circuits for the committing stage and $\left(O^{A}, O^{B}\right)$ is a pair of interactive quantum circuits for the opening stage (i.e. $A$ being the committer and $B$ the receiver). The committing stage generates the state $\left|\Psi_{b}\right\rangle=\left(C^{A} \odot C^{B}\right)|b\rangle^{A}|0\rangle^{B}$ upon which the opening stage is executed: $\left|\Psi_{\text {final }}\right\rangle=\left(O^{A} \odot O^{B}\right)\left|\Psi_{b}\right\rangle$. The binding condition of a quantum bit commitment is slightly more general than the usual classical definition. An adversary $\tilde{A}=\left(C^{\tilde{A}}, O^{\tilde{A}}\right)$ is such that $|\tilde{\Psi}\rangle=\left(C^{\tilde{A}} \odot C^{B}\right)|0\rangle^{\tilde{A}}|0\rangle^{B}$ is generated during the committing stage. The dishonest opening circuit $O^{\tilde{A}}$ tries to open $b \in\{0,1\}$ given as an extra input bit $|b\rangle^{\tilde{A}}$. Given the final state $\left|\tilde{\Psi}_{\text {final }}\right\rangle=\left(O^{\tilde{A}} \odot O^{B}\right)|b\rangle^{\tilde{A}}|\tilde{\Psi}\rangle$ we define $s_{b}(n)$ as the probability to open $b$ with success. More precisely, $s_{b}(n)=\| \mathbb{P}_{O K, b}^{B}\left|\tilde{\Psi}_{\text {final }}\right\rangle \|^{2}$ where $\mathbb{P}_{O K, b}^{B}$ is Bob's projection operator on the subspace leading to accept the opening of $b$. An adversary $\tilde{A}$ of the binding condition who can open $b=0$ with probability at least $s_{0}(n)$ and open $b=1$ with probability at least $s_{1}(n)$ will be called a $\left(s_{0}(n), s_{1}(n)\right)$-adversary against the binding condition. We define the concealing and binding criteria similarly to [6] : 
(computationally) binding: There exists no positive polynomial $p(n)$ and quantum $\left(s_{0}(n), s_{1}(n)\right)$-adversary $\tilde{A}$ such that $s_{0}(n)+s_{1}(n) \geq 1+\frac{1}{p(n)}$ for $n$ sufficiently large. The scheme is computationally binding if we add the restriction that $\|\tilde{A}\|_{\mathcal{U G}} \in \operatorname{poly}(n)$.

(computationally) concealing: For every interactive quantum circuit $\tilde{C}^{B}$ for the committing stage, all quantum circuits $L^{\tilde{B}}$ acting only upon $\tilde{B}$ 's registers, all positive polynomials $p(n)$ and $n$ sufficiently large, $\mathrm{P}\left(L^{\tilde{B}}\left(\left(C^{A} \odot C^{\tilde{B}}\right)|b\rangle^{A}|0\rangle^{\tilde{B}}\right)=b\right)<\frac{1}{2}+\frac{1}{p(n)}$ where the probabilities are taken over $b \in_{R}\{0,1\}$. The scheme is computationally concealing if we add the restriction $\left\|C^{\tilde{B}}\right\|_{\mathcal{U G}}+\left\|L^{\tilde{B}}\right\|_{\mathcal{U G}} \in \operatorname{poly}(n)$.

Note that the concealing and binding conditions are statistical not perfect.

Quantum Oblivious Transfer. A 1-2 quantum oblivious transfer protocol [5] involves a sender Alice holding input bits $\left(b_{0}, b_{1}\right)$ and a receiver Bob holding input $c \in\{0,1\}$. Alice sends $\left(b_{0}, b_{1}\right)$ to Bob in such a way that Bob receives only $b_{c}$ and Alice does not get to know $c$. The receiver must not be able to find $b_{\bar{c}}$ for at at least one $\bar{c} \in\{0,1\}$ and even given $b_{c}$. More precisely, a protocol $(A, B)$ for 1-2 quantum oblivious is such that $\left|\Psi\left(b_{0}, b_{1}, c\right)\right\rangle=(A \odot B)\left|b_{0} b_{1}\right\rangle^{A}|c\rangle^{B}$ allows Bob to recover $b_{c}$ from applying $\mathcal{M}_{+}$upon one of his registers. A protocol for 1-2 quantum oblivious transfer is (computationally) secure if it is both

(computationally) secure against the sender: For every quantum sender $\tilde{A}$, all quantum circuit $L^{\tilde{A}}$ acting only on $\tilde{A}$ 's registers, all positive polynomials $p(n)$ and $n$ sufficiently large, $\mathrm{P}\left(L^{\tilde{A}}\left((\tilde{A} \odot B)|00\rangle^{\tilde{A}}|c\rangle^{B}\right)=c\right)<\frac{1}{2}+\frac{1}{p(n)}$ where the probabilities are taken over $c \in_{R}\{0,1\}$. The security is computational if we add the restriction $\left\|L^{\tilde{A}}\right\|_{\mathcal{U G}}+\|\tilde{A}\|_{\mathcal{U G}} \in \operatorname{poly}(n)$.

(computationally) secure against the receiver: For every quantum receiver $\tilde{B}$, all quantum circuits $L^{\tilde{B}}$ acting only on $\tilde{B}$ 's registers, all positive polynomials $p(n)$ and $n$ sufficiently large, there exists a random variable $c$ with possible outcome 0 or 1 depending on $(A \odot \tilde{B})\left|b_{0} b_{1}\right\rangle^{A}|0\rangle^{\tilde{B}}$ satisfying $\mathrm{P}\left(L^{\tilde{B}}\left((A \odot \tilde{B})\left|b_{0} b_{1}\right\rangle^{A}|0\rangle^{\tilde{B}},\left|b_{c}\right\rangle^{\tilde{B}}\right)=b_{\bar{c}}\right)<\frac{1}{2}+\frac{1}{p(n)}$ where the probabilities are taken over $b_{0}, b_{1} \in_{R}\{0,1\}$. The security is computational if we add the restriction $\|\tilde{B}\|_{\mathcal{U G}}+\left\|L^{\tilde{B}}\right\|_{\mathcal{U G}} \in \operatorname{poly}(n)$.

As for bit commitment, the security is statistical not perfect.

\section{The Protocols}

In this section, we first describe the QOT protocol of [5] for 1-2 oblivious transfer. Then, we describe a simple quantum bit commitment scheme QBC, using QOT as a sub-protocol, that transforms any binding bit commitment scheme into a concealing one. Throughout this paper, we assume for simplicity that quantum transmission is error-free. 


\subsection{QOT Protocol}

The QOT protocol [5] is based upon the BB84 quantum coding scheme [1]. If the receiver (Bob) of a random BB84 qubit $|s\rangle_{\beta}, s \in_{R}\{0,1\}, \beta \in_{R}\{+, \times\}$ measures it in basis $\hat{\beta} \in_{R}\{+, \times\}$ upon reception, then a noisy classical communication of bit $s$ from Alice to Bob is implemented. Moreover, if later on Alice announces $\beta$, then Bob knows that he received $s$ whenever $\beta=\hat{\beta}$ and an uncorrelated bit whenever $\beta \neq \hat{\beta}$. The QOT protocol amplifies this process in order to get a secure 1-2 oblivious transfer. In order to ensure that Bob measures the BB84 qubits upon reception, bit commitments are used. Bob commits upon each measurement basis 11 and measurement outcome right after the quantum transmission. Alice then verifies in random positions that Bob has really measured the transmitted qubits by testing that whenever $\beta=\hat{\beta}$ then Bob's classical outcome $r \in\{0,1\}$ is such that $r=s$.

In the following, we assume that Alice and Bob have access to some bit commitment scheme BBC in order for Bob to commit upon the measurement bases of the received qubits together with the outcomes. Since the two commitments are made together, we write $\operatorname{BBC}(x, y)$ where $x \in\{+, \times\}$ and $y \in\{0,1\}$ for the commitments of both the measurement basis and the measurement outcome. BBC may be given as a black-box for bit commitment or may be provided from

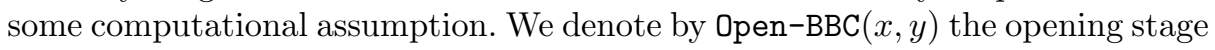
of $\operatorname{BBC}(x, y)$. Protocol $\mathrm{QOT}\left(b_{0}, b_{1}\right)(c)$ achieves the oblivious transfer of bit $b_{c}$.

\section{Protocol $1\left(\mathrm{QOT}\left(b_{0}, b_{1}\right)(c)\right)$}

1: For $1 \leq i \leq 2 n$

- Alice picks $s_{i} \in_{R}\{0,1\}, \beta_{i} \in_{R}\{+, \times\}$

- Alice sends to Bob a qubit $\pi_{i}$ in state $\left|s_{i}\right\rangle_{\beta_{i}}$

- Bob picks a basis $\hat{\beta}_{i} \in_{R}\{+, \times\}$, measures $\pi_{i}$ in basis $\hat{\beta}_{i}$, and obtains the outcome $r_{i} \in\{0,1\}$

2: For $1 \leq i \leq n$

- Bob runs $\operatorname{BBC}\left(\hat{\beta}_{i}, r_{i}\right)$ and $\operatorname{BBC}\left(\hat{\beta}_{n+i}, r_{n+i}\right)$ with Alice

- Alice picks $f_{i} \in_{R}\{0,1\}$ and announces it to Bob

- Bob runs Open-BBC $\left(\hat{\beta}_{n f_{i}+i}, r_{n f_{i}+i}\right)$

- Alice verifies that $\beta_{n f_{i}+i}=\hat{\beta}_{n f_{i}+i} \Rightarrow s_{n f_{i}+i}=r_{n f_{i}+i}$, otherwise she rejects the current execution

- if $f_{i}=0$ then Alice sets $\beta_{i} \leftarrow \beta_{n+i}$ and $s_{i} \leftarrow s_{n+i}$ and Bob sets $\hat{\beta}_{i} \leftarrow \hat{\beta}_{n+i}$ and $r_{i} \leftarrow r_{n+i}$

3: Alice announces her choices of bases $\beta_{1}, \beta_{2}, \ldots, \beta_{n}$ to Bob

4: Bob chooses at random and announces two subsets of positions $J_{0}, J_{1} \subset$ $\{1,2, \ldots, n\},\left|J_{0}\right|=\left|J_{1}\right|=\frac{n}{3}, J_{0} \cap J_{1}=\emptyset$, and $\forall i \in J_{c}, \beta_{i}=\hat{\beta}_{i}$.

5: Alice computes and announces $\hat{b}_{0}=\bigoplus_{j \in J_{0}} s_{j} \oplus b_{0}$ and $\hat{b}_{1}=\bigoplus_{j \in J_{1}} s_{j} \oplus b_{1}$

6: Bob receives $\left\langle\hat{b}_{0}, \hat{b}_{1}\right\rangle$ and computes $b_{c}=\bigoplus_{i \in J_{c}} r_{i} \oplus \hat{b}_{c}$

\footnotetext{
$\overline{1}$ The bases $\{+, \times\}$ are encoded in $\{0,1\}$.
} 
Known Security Results. The correctness and the security of the QOT protocol against the sender (Alice) has been reduced to the concealing property of $\mathrm{BBC}$ in [5]. The security against the receiver (Bob) has been provided by Yao in [19] given the commitment scheme BBC is perfectly binding. That is, given BBC is a perfect black-box for bit commitment then QOT is secure against any dishonest Bob irrespectively of his computing power.

\subsection{QBC Protocol Using QOT}

Given a binding but computationally concealing bit commitment scheme BBC in QOT the following simple commitment scheme will be shown concealing and computationally binding.

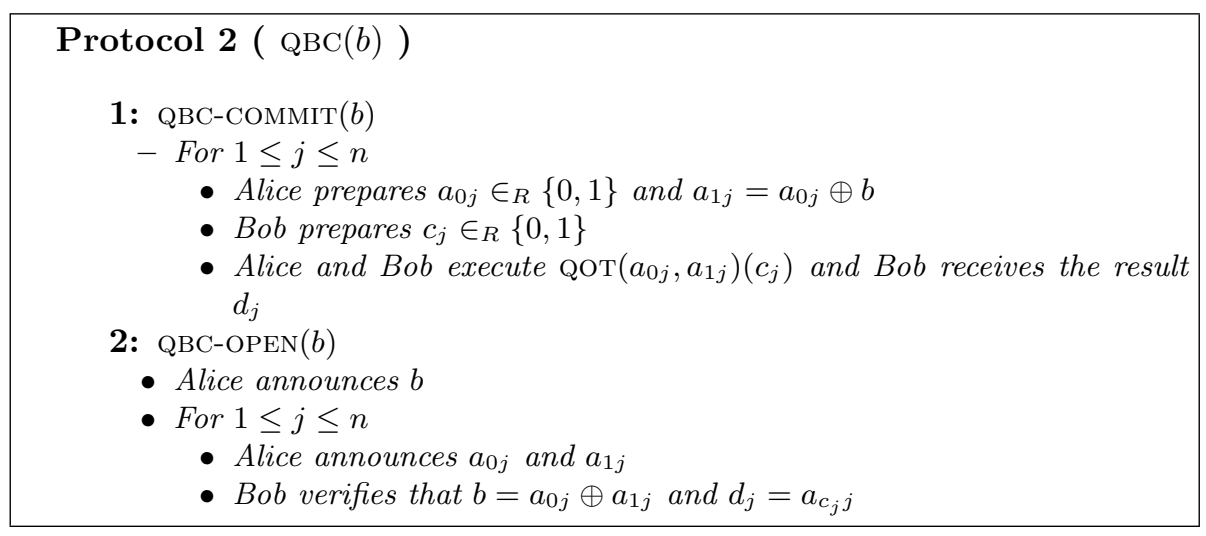

A commitment to bit $b$ is done by sending through 1-2 oblivious transfers $n$ pairs of bits $\left\{\left(a_{0 j}, a_{1 j}\right)\right\}_{j=1}^{n}$ such that $a_{0 j} \oplus a_{1 j}=b$. The concealing condition relies on the security of QOT against a malicious receiver and the binding condition relies on the security against a malicious sender. Intuitively, QBC appears concealing since for $1 \leq j \leq n$ Bob cannot obtain information on more than one of the two bits $\left(a_{0 j}, a_{1 j}\right)$ input in the $j$-th QOT and so, cannot determine $b=a_{0 j} \oplus a_{1 j}$. Similarly, QBC should be binding since for all $1 \leq j \leq n$ Alice needs to change the bit $a_{\bar{d}_{j} j}$ not selected by Bob in order to change her commitment.

More Notations. In the following we shall have to identify the variables generated during all calls to QOT in QBC. For that purpose, we use the following notation:

$-\pi_{i}^{j}$ is the $i$-th qubit sent in the $j$-th call to QOT in QBC.

$-\beta_{i}^{j} \in\{+, \times\}$ is the basis $\beta_{i}$ announced by Alice in the $j^{\text {th }}$ run of QOT in QBC. Note that a malicious Alice can send $\pi_{i}^{j}$ other than $|0\rangle_{\beta_{i}^{j}}$ and $|1\rangle_{\beta_{i}^{j}}$.

$-\hat{\beta}_{i}^{j} \in\{+, \times\}$ is the basis used by Bob to measure $\pi_{i}^{j}$ in the $j$-th call to QOT.

$-r_{i}^{j} \in\{0,1\}$ is the outcome of Bob's measurement of $\pi_{i}^{j}$ in basis $\hat{\beta}_{i}^{j}$.

- $\hat{r}_{i}^{j} \in\{0,1\}$ is Carl's outcome for measurement of $\pi_{i}^{j}$ in basis $\beta_{i}^{j}$.

- $J^{j}=\left(J_{0}^{j}, J_{1}^{j}\right)$ is the pair of sets announced by Bob in the $j^{\text {th }}$ run of QOT. 
We denote by bold lowercases the values for all executions at one glance: $\boldsymbol{\beta}=$ $\left\{\beta_{i}^{j}\right\}_{i, j}, \hat{\boldsymbol{\beta}}=\left\{\hat{\beta}_{i}^{j}\right\}_{i, j}, \boldsymbol{r}=\left\{r_{i}^{j}\right\}_{i, j}$, and $\hat{\boldsymbol{r}}=\left\{\hat{r}_{i}^{j}\right\}_{i, j}$. We denote by $\hat{\boldsymbol{b}}_{0}=\hat{b}_{0}^{1}, \ldots, \hat{b}_{0}^{n}$ and $\hat{\boldsymbol{b}}_{1}=\hat{b}_{1}^{1}, \ldots, \hat{b}_{1}^{n}$ the bits announced by Alice at step 5 of each call to QOT. Similarly, we denote by $\boldsymbol{a}=\left(\boldsymbol{a}_{0}, \boldsymbol{a}_{1}\right)=\left(a_{01}, a_{11}\right),\left(a_{02}, a_{12}\right), \ldots,\left(a_{0 n}, a_{1 n}\right) \in\{0,1\}^{2 n}$ Alice's announcements during the opening stage. We also denote $\boldsymbol{J}_{0}=J_{0}^{1}, \ldots, J_{0}^{n}$ and $\boldsymbol{J}_{1}=J_{1}^{1}, \ldots, J_{1}^{n}$ all sets announced by Bob and we write $\boldsymbol{J}=\left(\boldsymbol{J}_{0}, \boldsymbol{J}_{1}\right)$. Let $\boldsymbol{c}=c_{1}, \ldots, c_{n}$ be all selection bits used by Bob and let $\boldsymbol{d}=d_{1}, \ldots, d_{n}$ be all bits received by QOT. We write $\boldsymbol{J}_{c}=J_{c_{1}}^{1}, J_{c_{2}}^{2}, \ldots, J_{c_{n}}^{n}$ for all set of positions corresponding to qubits measured by Bob in bases announced by Alice.

\section{The Binding Condition}

In the following section, we show that $\mathrm{QBC}$ is secure against any Alice (the sender) who cannot break the concealing condition of the initial commitment scheme BBC. BBC is used in the calls to QOT in order for Bob to commit on his measurements and outcomes.

Simplified Version of QOT. In our analysis of the binding condition of QBC, we shall assume that the opening of half of the commitments in step 2 of QOT doesn't occur. The opening of the commitments allows Alice to make sure that Bob measured the qubits received in QOT upon reception. This test is not relevant to the binding condition of QBC.

Protocol $3\left(\mathrm{QOT}^{*}\left(b_{0}, b_{1}\right)(c)\right)$

1: ...step 1 of protocol 2

2: For $1 \leq i \leq n$

- Bob runs $\operatorname{BBC}\left(\hat{\beta}_{i}, r_{i}\right)$ and $\operatorname{BBC}\left(\hat{\beta}_{n+i}, r_{n+i}\right)$ with Alice

- Alice picks $f_{i} \in_{R}\{0,1\}$ and announces it to Bob

- if $f_{i}=0$ then Alice sets $\beta_{i} \leftarrow \beta_{n+i}$ and $s_{i} \leftarrow s_{n+i}$ and Bob sets $\hat{\beta}_{i} \leftarrow \hat{\beta}_{n+i}$ and $r_{i} \leftarrow r_{n+i}$

3-6: ... as steps 3 to 6 in protocol 2.

We omit the proof of the following simple lemma:

Lemma 3. If $\mathrm{QOT}^{*}$ is secure against the sender then QOT is secure against the sender.

Throughout Sect. 4, we shall assume implicitely calls to QOT* in QBC instead of calls to QOT. This simplifies the analysis and according to Lemma 3, it can be done without loss of generality.

\subsection{How to Prove the Binding Condition}

In order to show that QBC is computationally binding, we introduce intermediary protocols that will allow us to bridge the security of QBC with the known security 
of QOT given black-boxes for bit commitments. Let's consider the following four modified protocols:

U-QOT: Protocol QOT except that in step 2, Bob commits to random values. In other words, for $1 \leq i \leq n$, $\operatorname{Bob}$ runs $\operatorname{BBC}\left(u_{0 i}, u_{1 i}\right)$ and $\operatorname{BBC}\left(u_{2 i}, u_{3 i}\right)$ with $u_{0 i}, u_{2 i} \in_{R}\{+, \times\}$ and $u_{1 i}, u_{3 i} \in_{R}\{0,1\}$.

M-QOT: The same as U-QOT but a third party named Carl, for $1 \leq i \leq n$, intercepts the $i$-th qubit $\pi_{i}$ sent by Alice in step 1, measures in basis $\beta_{i}$ (announced by Alice in step 3) and sends the resulting state to Bob.

U-QBC: Protocol QBC using U-QOT.

$\mathrm{M}-\mathrm{QBC}$ : Protocol QBC using M-QOT.

The security against any dishonest sender in U-QOT and M-QOT is a direct consequence of the analysis in [5]. Since the commitments upon measurements do not carry any information about Bob's measurement, Alice cannot obtain any information about his selection bit $c$. The security is information-theoretic, no complexity assumption on Alice's computing power is required.

We reduce the security of the binding condition of $\mathrm{QBC}$ to the security of the concealing condition of BBC in two steps:

1. Using Lemmas 4 and 5, we conclude in Lemma 6 that U-QBC is binding. The modified protocol $\mathrm{M}-\mathrm{QBC}$ is used for reducing the security of U-QBC to the security of U-QOT. Carl's presence allows one to reduce the analysis to an essentially classical argument which becomes simpler than working from $\mathrm{U}-\mathrm{QBC}$ directly.

2. Theorem 1 establishes the desired result using the fact that an adversary for the binding condition of QBC cannot be an adversary of U-QBC (Lemma 6). It is shown how to construct an adversary for the concealing condition of $\mathrm{BBC}$ given an adversary for the binding condition of $\mathrm{QBC}$.

\subsection{U-QBC Is Binding}

In this section, we show that U-QBC is binding (Lemma 6) using Lemmas 4 and 5 as intermediary steps.

First, we show that an adversary against the binding condition of U-QBC can be transformed into an adversary against the binding condition of M-QBC.

Lemma 4. If there exists a $\left(s_{0}(n), s_{1}(n)\right)$-adversary $\tilde{A}$ against the binding condition of $\mathrm{U}-\mathrm{QBC}$ there also exists a $\left(s_{0}(n), s_{1}(n)\right)$-adversary $A^{*}$ against the binding condition of $\mathrm{M}-\mathrm{QBC}$.

Proof. We observe first that $\tilde{A}$ 's announcement of $\boldsymbol{\beta}$ at step 3 of U-QOT commutes with step 2 . That is, since only commitments to random values are received, $\tilde{A}$ can determine $\boldsymbol{\beta}$ without Bob's commitments. Moreover, $\tilde{A}$ could simulate the commitments on her own and then determine $\boldsymbol{\beta}$ before the qubits are sent to Bob at step 1 . Let $A^{*}$ be the quantum adversary that does that. If $\tilde{A}$ provides a $\left(s_{0}(n), s_{1}(n)\right)$-advantage in U-QBC then so it is for $A^{*}$. We now show that $A^{*}$ is also an adversary for the binding condition of M-QBC. 
Now assume for simplicity and without loss of generality that, Bob in UQBC or Bob and Carl in M-QBC wait until after Alice announces $\boldsymbol{a}=\left(\boldsymbol{a}_{0}, \boldsymbol{a}_{1}\right)$ before measuring all qubits received. It is easy to verify that this can always be done since nothing in the committing stage of $\mathrm{U}-\mathrm{QBC}$ or $\mathrm{M}-\mathrm{QBC}$ relies on those measurements' outcomes (i.e. since the commitments are made to random values). Clearly, postponing measurements do not influence Alice's probability of success at the opening stage.

Let $V=\left(\boldsymbol{\beta}, \boldsymbol{J}, \hat{\boldsymbol{b}}_{0}, \hat{\boldsymbol{b}}_{1}, \boldsymbol{c}, \boldsymbol{a}\right)$ be the partial view in U-QBC or in M-QBC up to Alice's announcement of $\boldsymbol{a}$ (and $b$ since for all $1 \leq j \leq n, a_{j 0} \oplus a_{j 1}=b$ ) in the opening stage. Let $\boldsymbol{V}_{\mathrm{U}}$ and $\boldsymbol{V}_{\mathrm{M}}$ be the random variable for the partial view in U-QBC and $\mathrm{M}-\mathrm{QBC}$ respectively. By construction we have that for all $V=\left(\boldsymbol{\beta}, \boldsymbol{J}, \hat{\boldsymbol{b}}_{0}, \hat{\boldsymbol{b}}_{1}, \boldsymbol{c}, \boldsymbol{a}\right), p(V)=\mathrm{P}\left(\boldsymbol{V}_{\mathrm{U}}=V\right)=\mathrm{P}\left(\boldsymbol{V}_{\mathrm{M}}=V\right)$. Moreover, we have that for all partial views $V$, the joint states $\left|\Psi_{\mathrm{U}}(V)\right\rangle$ for U-QBC and $\left|\Psi_{\mathrm{M}}(V)\right\rangle$ for M-QBC satisfy $\left|\Psi_{\mathrm{U}}(V)\right\rangle=\left|\Psi_{\mathrm{M}}(V)\right\rangle$. Let $\mathcal{V}_{b}=\left\{\left(\boldsymbol{\beta}, \boldsymbol{J}, \hat{\boldsymbol{b}}_{0}, \hat{\boldsymbol{b}}_{1}, \boldsymbol{c}, \boldsymbol{a}\right) \mid(\forall 1 \leq j \leq\right.$ $\left.n)\left[a_{j 0} \oplus a_{j 1}=b\right]\right\}$ be the set of partial views corresponding for Alice to open bit $b$. Given $V$, Bob's test will succeed if he gets $\boldsymbol{d}=\boldsymbol{a}_{\boldsymbol{c}}=a_{1 c_{1}}, a_{2 c_{2}}, \ldots, a_{n c_{n}}$ after measuring the qubits in positions in $\boldsymbol{J}_{\boldsymbol{c}}$ using Alice's bases $\beta_{i}^{j}$ for all $i \in J_{c_{j}}^{j}$ and $j \in\{1, \ldots, n\}$. Let $\mathcal{M}_{\text {test }}(V)=\left\{\mathbb{Q}_{o k}^{V}, \mathbb{1}-\mathbb{Q}_{o k}^{V}\right\}$ be the measurement allowing Bob to test Alice's announcement when she unveils $b$ given partial view $V \in \mathcal{V}_{b}$. $\mathbb{Q}_{o k}^{V}$ is the projection for the state of all qubits received in positions in $\boldsymbol{J}_{\boldsymbol{c}}$ into the subspace corresponding to parity $d_{j}=a_{j c_{j}}$ for all $j \in\{1, \ldots, n\}$. More precisely, $\mathbb{Q}_{o k}^{V}=\bigotimes_{j=1}^{n} \sum_{x \in T(V, j)} \mathbb{P}_{x}^{\boldsymbol{\beta}(V, j)}$ where $T(V, j)=\left\{x \in\{0,1\}^{\left|J_{c_{j}}^{j}\right|} \mid \oplus_{i}\right.$ $\left.x_{i}=a_{j c_{j}} \oplus \hat{b}_{c_{j}}^{j}\right\}$ and $\boldsymbol{\beta}(V, j)=\left\{\beta_{i}^{j} \mid i \in J_{c_{j}}^{j}\right\}$ for all $j \in\{1, \ldots, n\}$. Let $s_{b}^{\prime}(n)$ be the probability of success when $A^{*}$ opens $b$ in M-QBC. We get that

$$
s_{b}(n)=\sum_{V \in \mathcal{V}_{b}} p(V) \| \mathbb{Q}_{o k}^{V}\left|\Psi_{\mathrm{U}}(V)\right\rangle\left\|^{2}=\sum_{V \in \mathcal{V}_{b}} p(V)\right\| \mathbb{Q}_{o k}^{V} \mathbb{Q}_{o k}^{V}\left|\Psi_{\mathrm{M}}(V)\right\rangle \|^{2}=s_{b}^{\prime}(n)
$$

since the only difference between $\mathrm{U}-\mathrm{QBC}$ and $\mathrm{M}-\mathrm{QBC}$ is that in the former case both Carl and Bob measure the qubits in positions in $\boldsymbol{J}_{\boldsymbol{c}}$ with the same measurement $\mathcal{M}_{\text {test }}$ (this is why we have $\mathbb{Q}_{o k}^{V} \mathbb{Q}_{o k}^{V}=\mathbb{Q}_{o k}^{V}$ in (2) ). Carl's measurements for positions in $\boldsymbol{J}_{\bar{c}}$ are irrelevant to the success probability. The result follows.

Next, we reduce the binding condition of M-QBC to the security against the sender in M-QOT. We show that from any successful adversary against the binding condition of M-QBC one can construct an adversary able to extract non-negligible information about Bob's selection bit in M-QOT. Carl's measurements in M-QBC allows one to use a classical argument for most of the reduction thus simplifying the proof that $\mathrm{U}-\mathrm{QBC}$ is binding.

Lemma 5. If there exists a $\left(s_{0}(n), s_{1}(n)\right)$-adversary $\tilde{A}=\left(C^{\tilde{A}}, O^{\tilde{A}}\right)$ against the binding condition of $\mathrm{M}-\mathrm{QBC}$ with $s_{0}(n)+s_{1}(n) \geq 1+\frac{1}{p(n)}$ for some positive polynomial $p(n)$, then there also exists a cheating sender $A^{*}$ for M-QOT.

Proof. Let $a_{j 0}^{\prime}$ and $a_{j 1}^{\prime}$ be the two input bits for the $j$-th call to M-QOT computed according to Carl's outcomes $\hat{\boldsymbol{r}}$. Let $\boldsymbol{V}$ be the random variable for the joint view 
$\left(\boldsymbol{a}, \boldsymbol{a}^{\prime}, \boldsymbol{d}, \boldsymbol{c}\right)$ for an execution of the committing and the opening stages of MQBC between $\tilde{A}$ and an honest receiver $B$ and where $\tilde{A}$ is opening a random bit $b \in_{R}\{0,1\}$. Without loss of generality, we assume the announcements made by $\tilde{A}$ to be consistent, that is $a_{0 i} \oplus a_{1 i}=b$ for $1 \leq i \leq n$ when she opens bit $b$. Given $V=\left(\boldsymbol{a}, \boldsymbol{a}^{\prime}, \boldsymbol{d}, \boldsymbol{c}\right)$, we define the ordered set $S(V)=\left\{j \mid a_{j 0}^{\prime} \oplus a_{j 1}^{\prime} \neq a_{j 0} \oplus a_{j 1}\right\} \subseteq$ $\{1, \ldots, n\}$ of calls to M-QOT for which given view $V$ Alice's announcement of $\boldsymbol{a}$ disagree with Carl's outcomes $\boldsymbol{a}^{\prime}$. Given the ordered set $S(V)=\left\{\sigma_{1}, \sigma_{2}, \ldots, \sigma_{s}\right\}$, let $X_{j}(V) \in\{0,1\}$ for $1 \leq j \leq s$ be defined as

$$
X_{j}(V)=\left\{\begin{array}{l}
0 \text { if } d_{\sigma_{j}} \neq a_{\sigma_{j} c_{\sigma_{j}}} \\
1 \text { if } d_{\sigma_{j}}=a_{\sigma_{j} c_{\sigma_{j}}}
\end{array}\right.
$$

We let $X(V)=X_{1}(V), \ldots, X_{l(V)}(V)$ for $l(V)=\min \left(|S(V)|,\left\lceil\frac{n}{2}\right\rceil\right)$. Clearly, for $\tilde{A}$ to open with success given $V$, we must have $X(V)=1^{l(V)}$. Note that $\mathrm{P}\left(|S(\boldsymbol{V})| \geq \frac{n}{2}\right) \geq \frac{1}{2}$ since for at least one choice of $b,|S(\boldsymbol{V})| \geq \frac{n}{2}$ given that $\boldsymbol{V}$ always describes a consistent opening. We easily get that

$$
\begin{aligned}
\mathrm{P}(X(\boldsymbol{V})= & \left.1^{\left\lceil\frac{n}{2}\right\rceil}\right)=\mathrm{P}\left(X(\boldsymbol{V})=1^{l(\boldsymbol{V})}\right)-\mathrm{P}\left(X(\boldsymbol{V})=1^{l(\boldsymbol{V})} \wedge l(\boldsymbol{V})<\frac{n}{2}\right) \\
& \geq \frac{1}{2}\left(s_{0}(n)+s_{1}(n)\right)-\frac{1}{2} \mathrm{P}\left(X(\boldsymbol{V})=1^{l(\boldsymbol{V})} \mid l(\boldsymbol{V})<\frac{n}{2}\right) \geq \frac{1}{2 p(n)}
\end{aligned}
$$

Since $\sum_{x \in\{0,1\}^{\left\lceil\frac{n}{2}\right\rceil}} \mathrm{P}(X(\boldsymbol{V})=x) \leq 1$, for $n$ sufficiently large there exists a string $\hat{y}^{0} \in\{0,1\}^{\left\lceil\frac{n}{2}\right\rceil}$ such that $\mathrm{P}\left(X(\boldsymbol{V})=\hat{y}^{0}\right) \leq \frac{1}{4 p(n)}$. Let $\rho$ be the number of zeros in $\hat{y}^{0}$ and $R\left(\hat{y}^{0}\right)=\left\{r_{1}, r_{2}, \ldots, r_{\rho}\right\} \subseteq\left\{1, \ldots,\left\lceil\frac{n}{2}\right\rceil\right\}$ be the ordered set of positions $1 \leq r \leq\left\lceil\frac{n}{2}\right\rceil$ where $\hat{y}_{r}^{0}=0$. We now define for $1 \leq j \leq \rho$ the hybrid strings $\hat{y}^{j}=\hat{y}_{1}^{j} \hat{y}_{2}^{j} \ldots \hat{y}_{\left\lceil\frac{n}{2}\right\rceil}^{j}$ between $\hat{y}^{0}$ and $1^{\left\lceil\frac{n}{2}\right\rceil}$ :

$$
\hat{y}_{i}^{j}=\left\{\begin{array}{l}
1 \text { if } i=r_{k} \text { for } k \leq j \\
\hat{y}_{i}^{0} \text { Otherwise }
\end{array}\right.
$$

Hence, $\mathrm{P}\left(X(\boldsymbol{V})=\hat{y}^{\rho}=1^{n}\right)-\mathrm{P}\left(X(\boldsymbol{V})=\hat{y}^{0}\right) \geq \frac{1}{4 p(n)}$ and we conclude by an hybrid argument that there exist $1 \leq k^{*} \leq \rho$ such that

$$
\mathrm{P}\left(X(\boldsymbol{V})=\hat{y}^{k^{*}}\right)-\mathrm{P}\left(X(\boldsymbol{V})=\hat{y}^{k^{*}-1}\right) \geq \frac{1}{\rho 4 p(n)} \geq \frac{1}{2(n+1) p(n)}
$$

Note that $\hat{y}^{k^{*}}$ and $\hat{y}^{k^{*}-1}$ differs only by the bit in position $r_{k^{*}}$ where they respectively have a 1 and a 0 .

$A^{*}$ uses $\tilde{A}$ and $B=\left(C^{B}, O^{B}\right)$ in the following way: after choosing $h \in_{R}$ $\{1, \ldots, n\}$, it makes $\tilde{A}$ interact with a simulated honest receiver $B$ for $\mathrm{M}$-QBC except for the $h$-th execution of M-QOT for which $\tilde{A}$ interacts with the targeted receiver for M-QOT. Let $V=\left(\boldsymbol{a}, \boldsymbol{a}^{\prime}, \boldsymbol{d}, \boldsymbol{c}\right)$ be the view generated during the execution. Given $A^{*}$ 's view, algorithm $L^{A^{*}}$ produces a guess $\tilde{c}$ for Bob's selection bit $c=c_{h}$ in M-QOT as follows: 
- If $|S(V)| \geq\left\lceil\frac{n}{2}\right\rceil, h=\sigma_{r_{k^{*}}}$ and $\forall i \in\left\{1, \ldots,\left\lceil\frac{n}{2}\right\rceil\right\} \backslash\left\{r_{k^{*}}\right\}, X_{i}(V)=\hat{y}_{i}^{k^{*}}$, then $\tilde{c} \in\{0,1\}$ is defined such that $a_{h \tilde{c}}=a_{h \tilde{c}}^{\prime}$ (which necessarily exists since $h \in S(V))$,

- Otherwise, $\tilde{c} \in_{R}\{0,1\}$.

Let $\mathcal{T}(V)$ be the event of a successful test in the previous computation. Since independently $|S(V)| \geq \frac{n}{2}$ with probability at least $\frac{1}{2}, h=\sigma_{r_{k^{*}}}$ with probability $\frac{1}{n}$, and $\forall i \in\left\{1, \ldots,\left\lceil\frac{n}{2}\right\rceil\right\} \backslash\left\{r_{k^{*}}\right\}, X_{i}(V)=\hat{y}_{i}^{k^{*}}$ with probability $\mathrm{P}\left(X(\boldsymbol{V})=\hat{y}^{k^{*}}\right)+\mathrm{P}\left(X(\boldsymbol{V})=\hat{y}^{k^{*}-1}\right)$, we have that

$$
\mathrm{P}(\mathcal{T}(\boldsymbol{V})) \geq \frac{\mathrm{P}\left(X(\boldsymbol{V})=\hat{y}^{k^{*}}\right)+\mathrm{P}\left(X(\boldsymbol{V})=\hat{y}^{k^{*}-1}\right)}{2 n} .
$$

Given $\mathcal{T}(V)$, the guess $\tilde{c}$ is the only value for Bob's selection bit $c$ that would lead to $X(V)=\hat{y}^{k^{*}}$ instead of $X(V)=\hat{y}^{k^{*}-1}$ (the two strings are the only possible given $\mathcal{T}(V))$. We get that

$$
\mathrm{P}(\tilde{c}=c \mid \mathcal{T}(\boldsymbol{V}))=\frac{\mathrm{P}\left(X(\boldsymbol{V})=\hat{y}^{k^{*}}\right)}{\mathrm{P}\left(X(\boldsymbol{V})=\hat{y}^{k^{*}}\right)+\mathrm{P}\left(X(\boldsymbol{V})=\hat{y}^{k^{*}-1}\right)} .
$$

It follows that $\left(A^{*}, L^{A^{*}}\right)$ is a cheating sender for M-QOT since

$$
\begin{aligned}
\mathrm{P}(\tilde{c}=c) & =\frac{1}{2}(1-\mathrm{P}(\mathcal{T}(\boldsymbol{V})))+\mathrm{P}(\mathcal{T}(\boldsymbol{V})) \mathrm{P}(\tilde{c}=c \mid \mathcal{T}(\boldsymbol{V})) \\
& \geq \frac{1}{2}+\frac{1}{8 n(n+1) p(n)} .
\end{aligned}
$$

Using Lemmas 3, 4 and 5 together with the fact that M-QOT is unconditionally secure against the sender [5], we get the desired result:

Lemma 6. Protocol U-QBC is binding.

As we shall see next, Lemma [6 helps a great deal in proving that QBC is computationally binding.

\subsection{QBC Is Binding when BBC Is Concealing}

In the following, we conclude that $\mathrm{QBC}$ is computationally binding whenever $\mathrm{BBC}$ is computationally concealing. We use the fact that $\mathrm{U}-\mathrm{QBC}$ is binding (Lemma 6) in order to use any adversary against the binding condition of QBC as a distinguisher between random (U-QBC) and real (QBC) commitments for some hybrids between U-QBC and QBC.

Theorem 1. If there exists a $\left(s_{0}(n), s_{1}(n)\right)$-adversary $\tilde{A}=\left(C^{\tilde{A}}, O^{\tilde{A}}\right)$ against the binding condition of $\mathrm{QBC}$ with $s_{0}(n)+s_{1}(n) \geq 1+\frac{1}{p(n)}$ for a positive polynomial $p(n)$, then there exists a quantum receiver $C^{\tilde{B}}$ in $\mathrm{BBC}$ and a quantum algorithm $L^{\tilde{B}}$ such that $\mathrm{P}\left(L^{\tilde{B}}\left(\left(C^{A} \odot C^{\tilde{B}}\right)|b\rangle^{A}|0\rangle^{\tilde{B}}\right)=b\right) \geq \frac{1}{2}+\Omega\left(\frac{1}{n^{4} p(n)}\right)$ whenever $b \in_{R}\{0,1\}$ and where $C^{\tilde{B}}$ calls $\tilde{A}$ an expected $O\left(n^{5} p(n)^{2}\right)$ times. 
Proof. Let $B=\left(C^{B}, O^{B}\right)$ be the circuits for the honest receiver in QBC and let $\mathcal{A}$ be an honest committer in BBC. Given $\tilde{A}$, we construct a receiver $C^{\tilde{B}}$ in $\mathrm{BBC}$ from which a bias for $\mathcal{A}$ 's committed bit can be extracted. Remember that the only difference between $\mathrm{U}-\mathrm{QBC}$ and $\mathrm{QBC}$ is that a honest receiver commits to random bits instead of his measurements and outcomes. There are $4 n$ calls to Commit-BBC per QOT (U-QOT) for a total of $4 n^{2}$ during the committing stage of QBC (U-QBC). Let's note as significant the committed bits specified by the protocol QOT (to measurements and outcomes) and as random the ones specified by the protocol U-QOT (to random bits). We describe hybrids in between QBC and U-QBC by letting the number of significant and random commitments vary. Let $\mathrm{QBC}^{k}$ be protocol $\mathrm{QBC}$ but where the first $k$ commitments out of $4 n^{2}$ are made to random values. We have that $\mathrm{U}-\mathrm{QBC} \equiv \mathrm{QBC}^{4 n^{2}}$ is binding whereas $\tilde{A}$ is a $\left(s_{0}(n), s_{1}(n)\right)$-adversary for the binding condition of $\mathrm{QBC}^{0} \equiv \mathrm{QBC}$. Let $s_{b}^{k}(n)$ be the probability that $\tilde{A}$ succeeds when opening $b \in\{0,1\}$ in $\mathrm{QBC}^{k}$ for $0 \leq k \leq 4 n^{2}$. Defining $\hat{s}^{k}(n)=\left(s_{0}^{k}(n)+s_{1}^{k}(n)\right) / 2$, we get that $\hat{s}^{0}(n) \geq \frac{1}{2}+\frac{1}{2 p(n)}$ and from Lemma $6 \hat{s}^{4 n^{2}}(n)<\frac{1}{2}+\frac{1}{e(n)}$ where $e(n)>p(n)$ for all $p(n) \in \operatorname{poly}(n)$ and $n$ sufficiently large. By the hybrid argument, there exists $0 \leq k^{*} \leq 4 n^{2}-1$ such that for $n$ sufficiently large,

$$
\hat{s}^{k^{*}}(n)-\hat{s}^{k^{*}+1}(n) \geq \frac{1}{9 n^{2} p(n)} .
$$

Hence, $\mathcal{D}_{4 n^{2}}\left(\frac{1}{9 n^{2} p(n)}\right)=\left\{\hat{s}^{i}(n)\right\}_{i=0}^{4 n^{2}}$ is a family of Bernoulli distributions that satisfies the condition of Lemma 2 The sampling circuit $\mathrm{S}$ is easy to construct given $\tilde{A}$ and $B$. Upon classical input $|l\rangle$ for $0 \leq l \leq 4 n^{2}$, S runs $\tilde{A}$ and $B$ except that the first $l$ commitments sent from $B$ to $\tilde{A}$ (using BBC) are made to random values instead of the measurements $\hat{\boldsymbol{\beta}}$ and the outcomes $\boldsymbol{r}$. $\tilde{A}$ then opens a random bit $b \in_{R}\{0,1\}$. If $B$ accepts the opening of $b$ then $\mathrm{S}(|l\rangle)=1$ otherwise it returns $\mathrm{S}(|l\rangle)=0$. Circuit $\mathrm{S}$ is therefore a sampling circuit for $\mathcal{D}_{4 n^{2}}\left(\frac{1}{9 n^{2} p(n)}\right)$ such that $\|\mathbf{S}\|_{\mathcal{U G}} \in O\left(\|\tilde{A}\|_{\mathcal{U G}}\right)$ assuming without loss of generality that $\|B\|_{\mathcal{U G}} \in O\left(\|\tilde{A}\|_{\mathcal{U G}}\right)$.

We now construct the adversary $C^{\tilde{B}}$ for the concealing condition of BBC given $\tilde{A}$. In order to use algorithm FindDrop (defined in Sect. 2.1), $C^{\tilde{B}}$ must first determine a lower bound $\frac{1}{p^{\prime}(n)}$ for the drop $\frac{1}{9 n^{2} p(n)}$. This is done by finding a lower bound $\tilde{p}(n)$ for $\frac{1}{2 p(n)}$ and then setting $p^{\prime}(n)=\frac{5 n^{2}}{\tilde{p}(n)} \cdot C^{\tilde{B}}$ computes $\tilde{p}(n)=$ LowBound $\left(\mathrm{S}_{0}, \frac{1}{2}, n\right)$ (defined in Sect. 2.1) where $\mathrm{S}_{0}$ is the circuit $\mathrm{S}$ with the input bits fixed to $|0\rangle$. From Lemma 1, LowBound returns $\tilde{p}(n)$ such that $\frac{1}{2 n^{2} p(n)} \leq$ $\tilde{p}(n) \leq \frac{1}{2 p(n)}$ except with negligible probability and after an expected $O\left(n^{5} p(n)^{2}\right)$ calls to $\mathrm{S}_{0}$.

Now $C^{\tilde{B}}$ can use FindDrop $\left(\mathrm{S}, \frac{1}{p^{\prime}(n)}, n\right)$ with the family of distributions $\mathcal{D}_{4 n^{2}}\left(\frac{1}{p^{\prime}(n)}\right)=\left\{\hat{s}^{i}(n)\right\}_{i=0}^{4 n^{2}}$ which exhibits a drop $\frac{1}{p^{\prime}(n)}$ except with negligible probability. From Lemma 2, $C^{\tilde{B}}$ gets $0 \leq \kappa \leq 4 n^{2}-1$ such that

$$
\hat{s}^{\kappa}(n)-\hat{s}^{\kappa+1}(n) \geq \frac{1}{2 p^{\prime}(n)}
$$


except with negligible probability. The value of $\kappa$ is obtained after calling $\mathbf{S}$ (including the calls to $\mathrm{S}_{0}$ in LowBound) an expected $O\left(n^{5} p(n)^{2}\right)$ times.

$C^{\tilde{B}}$ then uses $\kappa$ for attacking the concealing condition of BBC in the following way: It makes $\tilde{A}$ and $B$ interact (where $\tilde{A}$ opens $b \in_{R}\{0,1\}$ ) as in $\mathrm{QBC}^{\kappa+1}$ except that the $(\kappa+1)$-th random commitment is provided by the committer $\mathcal{A}$ in BBC. Let $b \in\{0,1\}$ be the bit committed by $\mathcal{A}$. Let $\boldsymbol{V}$ be the random variable for the view generated during the interaction between $\tilde{A}$ and $B$ when $\tilde{A}$ opens the random bit. Let $c_{\kappa+1}(V) \in\{0,1\}$ be the bit that $B$ would have committed if the $(\kappa+1)$-th commitment was significant. The distinguisher $L^{\tilde{B}}$ (which is classical given the view $V$ ) returns the guess $\tilde{b}$ for $b$ the following way:

- If $V$ is a successful opening then $\tilde{b}=c_{\kappa+1}(V)$,

- Otherwise, $\tilde{b} \in_{R}\{0,1\}$.

Let $\mathcal{V}_{o k}^{\kappa+1}$ be the set of views for $\mathrm{QBC}^{\kappa+1}$ resulting in a successful opening and let $\mathcal{G}$ be the set of values $\kappa$ for which (9) holds. We have $\hat{s}^{\kappa}(n)=\mathrm{P}\left(\boldsymbol{V} \in \mathcal{V}_{o k}^{\kappa+1} \mid c_{\kappa+1}(\boldsymbol{V})=b\right)$ and $\hat{s}^{\kappa+1}(n)=$ $\frac{1}{2}\left(\mathrm{P}\left(\boldsymbol{V} \in \mathcal{V}_{o k}^{\kappa+1} \mid c_{\kappa+1}(\boldsymbol{V}) \neq b\right)+\mathrm{P}\left(\boldsymbol{V} \in \mathcal{V}_{o k}^{\kappa+1} \mid c_{\kappa+1}(\boldsymbol{V})=b\right)\right) \quad$ which, $\quad$ using (9), leads to

$$
\mathrm{P}\left(\boldsymbol{V} \in \mathcal{V}_{o k}^{\kappa+1} \wedge c_{\kappa+1}(\boldsymbol{V}) \neq b\right) \leq \mathrm{P}\left(\boldsymbol{V} \in \mathcal{V}_{o k}^{\kappa+1} \wedge c_{\kappa+1}(\boldsymbol{V})=b\right)-\frac{1}{2 p^{\prime}(n)}
$$

Since we also have $\mathrm{P}\left(\boldsymbol{V} \in \mathcal{V}_{o k}^{\kappa+1}\right)=\mathrm{P}\left(\boldsymbol{V} \in \mathcal{V}_{o k}^{\kappa+1} \wedge c_{\kappa+1}(\boldsymbol{V}) \neq b\right)+$ $\mathrm{P}\left(\boldsymbol{V} \in \mathcal{V}_{o k}^{\kappa+1} \wedge c_{\kappa+1}(\boldsymbol{V})=b\right)$, we get

$$
\begin{aligned}
\mathrm{P}(\tilde{b}=b \mid \kappa \in \mathcal{G}) & =\mathrm{P}\left(\boldsymbol{V} \in \mathcal{V}_{o k}^{\kappa+1} \wedge c_{\kappa+1}(\boldsymbol{V})=b\right)+\frac{1}{2}\left(1-\mathrm{P}\left(\boldsymbol{V} \in \mathcal{V}_{o k}^{\kappa+1}\right)\right) \\
& \geq \frac{1}{2}\left(1+\frac{1}{2 p^{\prime}(n)}\right)
\end{aligned}
$$

Since $\mathrm{P}(\tilde{b}=b) \geq \mathrm{P}(\kappa \in \mathcal{G}) \mathrm{P}(\tilde{b}=b \mid \kappa \in \mathcal{G})$ and $\mathrm{P}(\kappa \in \mathcal{G}) \geq 1-2^{-\alpha n}, \alpha>$ 0 (Lemma 1) we finally get that $\left(C^{\tilde{B}}, L^{\tilde{B}}\right)$ is an adversary for the concealing condition of BBC providing a bias in $\Omega\left(\frac{1}{p^{\prime}(n)}\right)=\Omega\left(\frac{1}{n^{4} p(n)}\right)$ after calling $\tilde{A}$ an expected $O\left(n^{5} p(n)^{2}\right)$ times.

\section{The Concealing Condition}

We now reduce the concealing condition of QBC to the security of QOT against a malicious receiver.

Lemma 7. If there exists a quantum circuit $C^{\tilde{B}}$ for the receiver in Commit-QBC and a quantum algorithm $L^{\tilde{B}}$ acting only on $\tilde{B}^{\prime}$ s registers such that $\mathrm{P}\left(L^{\tilde{B}}\left(\left(C^{A} \odot C^{\tilde{B}}\right)|b\rangle^{A}|0\rangle^{\tilde{B}}\right)=b\right) \geq \frac{1}{2}+\frac{1}{p(n)}$ for some positive polynomial $p(n)$ and an honest committing circuit $C^{A}$ for $b \in_{R}\{0,1\}$, then there also exists a cheating receiver $\left(B^{*}, L^{B^{*}}\right)$ for QOT. 
Proof. For the receiver $C^{\tilde{B}}$ and $C^{A}$ described in the statement, we have

$$
\begin{aligned}
\mathrm{P}\left(L^{\tilde{B}}\left(\left(C^{A} \odot C^{\tilde{B}}\right)|1\rangle^{A}|0\rangle^{\tilde{B}}\right)=\right. & 1)- \\
& \mathrm{P}\left(L^{\tilde{B}}\left(\left(C^{A} \odot C^{\tilde{B}}\right)|0\rangle^{A}|0\rangle^{\tilde{B}}\right)=1\right) \geq \frac{2}{p(n)} .
\end{aligned}
$$

Let's define a modification of an honest committing circuit for QBC, noted $C^{\tilde{A}}$, which is the same as $C^{A}$ but takes a string $\hat{f} \in\{0,1\}^{n}$ instead of a bit $b$ and sends in the $i$-th call to QOT the bits $a_{0 i} \in_{R}\{0,1\}$ and $a_{1 i}=a_{0 i} \oplus \hat{f}_{i}$ for $1 \leq i \leq n$. The circuit $C^{A}$ with input $b$ is equivalent to $C^{\tilde{A}}$ with input $b^{n}$. Once again, by an hybrid argument, there exists $1 \leq k^{*} \leq n$ such that for

$$
\begin{gathered}
\mathrm{P}\left(L^{\tilde{B}}\left(\left(C^{\tilde{A}} \odot C^{\tilde{B}}\right)\left|1^{k^{*}} 0^{n-k^{*}}\right\rangle^{\tilde{A}}|0\rangle^{\tilde{B}}\right)=1\right) \\
\mathrm{P}\left(L^{\tilde{B}}\left(\left(C^{\tilde{A}} \odot C^{\tilde{B}}\right)\left|1^{k^{*}-1} 0^{n-k^{*}+1}\right\rangle^{\tilde{A}}|0\rangle^{\tilde{B}}\right)=1\right) \\
\geq \frac{2}{n p(n)} .
\end{gathered}
$$

With such value $k^{*}, B^{*}$ cheats an honest sender $A^{\prime}$ for $\operatorname{QOT}\left(e_{0}, e_{1}\right)(0)$ in the following way: it makes $C^{\tilde{B}}$ interact with $C^{\tilde{A}}$ with input $\left(1^{k^{*}-1} ? 0^{n-k^{*}}\right)$ for Commit-QBC except for the $k^{*}$-th call to QOT where it makes $C^{\tilde{B}}$ interact with the targeted sender $A^{\prime}$ with inputs $e_{0}, e_{1} \in_{R}\{0,1\}$. Then, knowing $e_{c}$ for $c \in\{0,1\}$, we take the output of $L^{\tilde{B}}, b^{\prime}$ say, and compute a guess $e_{c} \oplus b^{\prime}$ for $e_{\bar{c}}$. For this algorithm $L^{B^{*}}$ we have

$$
\begin{aligned}
\mathrm{P}\left(L^{B^{*}}\left(\left(A^{\prime} \odot B^{*}\right)\left|e_{0} e_{1}\right\rangle^{A}|0\rangle^{B^{*}},\left|e_{c}\right\rangle^{B^{*}}\right)=e_{\bar{c}}\right)=\mathrm{P}\left(b^{\prime}=e_{0} \oplus e_{1}\right) & \\
& \geq \frac{1}{2}+\frac{1}{n p(n)}
\end{aligned}
$$

where the probabilities are taken over $e_{0}, e_{1} \in_{R}\{0,1\}$.

From Yao's result [19] and Lemma 7 it is straightforward to conclude that QBC is concealing.

\section{Conclusion and Open Questions}

Having shown in Theorem 1, that a computationally concealing BBC results in a computationally binding QBC and, from Lemma 7 together with Yao's result [19], that no adversary against the concealing condition of QBC exists, we conclude with our main result:

Theorem 2. If $\mathrm{BBC}$ is binding and computationally concealing then $\mathrm{QBC}$ is concealing and computationally binding. 
For security parameter $n$, the reduction of an adversary $\left(C_{n}^{\tilde{B}}, L_{n}^{\tilde{B}}\right)$ for the concealing condition of $\mathrm{BBC}$ to an adversary $\tilde{A}_{n}$ for the binding condition of $\mathrm{QBC}$ is expected polynomial-time black-box. The adversary $\left\{\left(C_{n}^{\tilde{B}}, L_{n}^{\tilde{B}}\right)\right\}_{n>0}$ is a uniform family of quantum circuits whenever $\left\{\tilde{A}_{n}\right\}_{n>0}$ is uniform. It is an interesting open problem to find an exact polynomial-time black-box reduction.

One consequence of Theorem 2 is that concealing commitment schemes can be built from any quantum one-way function. We first observe that Naor's commitment scheme [17] is also secure against the quantum computer if the pseudorandom bit generator (PRBG) it is based upon is secure against the quantum computer. This follows from the fact that any quantum circuit able to distinguish between commitments to 0 and 1 is also able to distinguish a truly random sequence from a pseudo-random one. To complete the argument, we must make sure that given a quantum one-way function one can construct a PRBG resistant to quantum distinguishers. A tedious but not difficult exercise allows to verify that the classical construction of [11] results in a PRBG secure against quantum distinguishers given it is built from quantum one-way functions. We get the following corollary which is not known to hold in the classical case:

Corollary 1. Both binding but computationally concealing and concealing but computationally binding quantum bit commitments can be constructed from quantum one-way functions.

It would be interesting to find a concealing quantum bit commitment scheme directly constructed from one-way functions which improves the complexity of our construction. Is it possible to find a non-interactive concealing commitment scheme from the same complexity assumption or are such constructions inherently interactive? It is also unclear whether or not perfectly concealing schemes can be based upon any quantum one-way function.

Although we assumed in this paper a perfect quantum channel, our construction should also work with noisy quantum transmission [3]. It would be nice to provide the analysis for this general case.

\section{References}

1. C. H. Bennett and G. Brassard. Quantum cryptography: Public key distribution and coin tossing. In Proceedings of IEEE International Conference on Computers, Systems, and Signal Processing, pages 175-179. IEEE, 1984.

2. Barenco, A., C. H. Bennett, R. Cleve, D. P. DiVincenzo, N. MargoLus, P. Shor, T. Sleator, J. Smolin and H. Weinfurter, "Elementary Gates for Quantum Computation", Physical Review A, vol. 52, no 5, November 1995, pp. $3457-3467$.

3. Bennett, C. H., G. Brassard, C. Crépeau and M.-H. Skubiszewska, "Practical Quantum Oblivious Transfer", Advances in Cryptology : CRYPTO '91 : Proceedings, Lecture Notes in Computer Science, vol. 576, Springer-Verlag, August 1992, pp. $362-371$.

4. Brassard, G., D. Chaum and C. Crépeau, "Minimum Disclosure Proofs of Knowledge", Journal of Computing and System Science, vol. 37 , 1988, pp. $156-189$. 
5. Crépeau, C., "Quantum Oblivious Transfer", Journal of Modern Optics, vol. 41, no 12 , December 1994, pp. $2445-2454$. A preliminary version of this work appeared in Crépeau, C. and J. Kilian, "Achieving oblivious transfer using weakened security assumptions", Proceedings of 29th IEEE Symposium on the Foundations of Computer Science, October 1988, pp. 42-52.

6. Dumais, P., D. Mayers, and L. Salvail, "Perfectly Concealing Quantum Bit Commitment From Any Quantum One-Way Permutation", Advances in Cryptology : EUROCRYPT '00 : Proceedings, Lecture Notes in Computer Science, vol. 1807, Springer-Verlag, 2000, pp. $300-315$.

7. Goldreich, O., and L. Levin, "A Hard-Core Predicate for Any One-Way Function", Proceedings of the 21st ACM Symposium on Theory of Computing, 1989, pp. $25-32$.

8. Goldwasser, S., S. Micali and C. Rackoff, "The Knowledge Complexity of Interactive Proof Systems", SIAM Journal on Computing, vol. 18, 1989, pp. $186-208$.

9. Goldreich, O., S. Micali, and A. Wigderson, "Proofs that Yield Nothing but their Validity or All Language in NP Have Zero-Knowledge Proof Systems", Journal of the $A C M$, vol. 38, no 1, 1991, pp. 691-729.

10. Goldreich, O., S. Micali, and A. Wigderson, "How to play any mental game or a completeness theorem for protocols with honest majority", Proceedings of the 19th ACM Symposium on Theory of Computing, 1987, pp. 218-229.

11. HÅstad, J., R. Impagliazzo, L. Levin and M. Luby "A pseudo-random generator from any one-way function", SIAM Journal on Computing, vol. 28, no 4, 1999, pp. $1364-1396$.

12. Impagliazzo, R. and S. Rudich, "Limits on Provable Consequences of OneWay Permutations", Advances in Cryptology : CRYPTO '88 : Proceedings, Lecture Notes in Computer Science, vol. 403, Springer-Verlag, 1989, pp. $2-7$.

13. LÉgaré, F., "Converting the flavor of a quantum bit commitment", M.Sc. thesis, School of Computer Science, McGill University, 2001. Supervised by C. Crépeau. Thesis available at http://www.cs.McGill.ca/ crepeau/students.html.

14. Lo, H.-K. and H. F. ChaU, "Is quantum Bit Commitment Really Possible?", Physical Review Letters, vol. 78, no 17, April 1997, pp. 3410-3413.

15. Mayers, D., "The Trouble With Quantum Bit Commitment", available at http://xxx.lanl.gov/abs/quant-ph/9603015.

16. Mayers, D., "Unconditionally Secure Quantum Bit Commitment is Impossible", Physical Review Letters, vol. 78, no 17, April 1997, pp. 3414-3417.

17. NAOR, M., "Bit Commitment Using Pseudo-Randomness", Journal of Cryptology, vol. 4, 1991, pp. $151-158$.

18. Naor, M., R. Ostrovsky, R. Ventkatesan, and M. Young, "Perfect ZeroKnowledge Arguments For NP Using Any One-Way Permutation", Journal of Cryptology, vol. 11, no 2, 1998, pp. 87-108.

19. YAO, A. C., "Security of Quantum Protocols Against Coherent Measurements", Proceedings of the 27th ACM Symposium on Theory of Computing, 1995, pp. $67-75$. 